\title{
Roughening transition in a model for dimer adsorption and desorption
}

\author{
Haye Hinrichsen ${ }^{1}$ and Géza Ódor ${ }^{2}$ \\ ${ }^{1}$ Max-Planck-Institut für Physik komplexer Systeme, Nöthnitzer Straße 38, 01187 Dresden, Germany \\ ${ }^{2}$ Research Institute for Technical Physics and Materials Science, P. O. Box 49, H-1525 Budapest, Hungary
}

(October 16, 1998)

\begin{abstract}
A solid-on-solid growth model for dimer adsorption and desorption is introduced and studied numerically. The special property of the model is that dimers can only desorb at the edges of terraces. It is shown that the model exhibits a roughening transition from a smooth to a rough phase. In both phases the interface remains pinned to the bottom layer and does not propagate. Close to the transition certain critical properties are related to those of a unidirectionally coupled hierarchy of parity-conserving branching-annihilating random walks.
\end{abstract}

PACS numbers: 64.35.Rh, 05.70.Ln, 82.20.Wt.

The study of crystal growth and transitions between different morphologies of moving interfaces is a field that continues to attract great interest [1]. Various models have been developed in order to describe the essential features of roughening transitions [2]. In many cases the critical properties close to the transition are universal, i.e., they do not depend on the microscopic details of the model. For example, the majority of roughening transitions in $d>2$ spatial dimensions belongs to the Kardar-Parisi-Zhang universality class [3]. Therefore it is an important theoretical task to categorize the possible universality classes for roughening transitions in a given geometry.

It is well known that one-dimensional interface models with short-range interactions at thermal equilibrium do not exhibit roughening transitions. However, under nonequilibrium conditions such a transition may occur even in one spatial dimension. Known examples include polynuclear growth (PNG) models [4], solid-onsolid models with evaporation at the edges of terraces [5], and certain models for fungal growth [6]. The key feature of all these growth processes is a close relationship to directed percolation (DP) [7] 8 . More precisely, the DP process emerges at a particular reference height of the interface. In the models of Ref. [5] this reference height is the spontaneously selected bottom layer of the interface, whereas in polynuclear and fungal models, which evolve by parallel dynamics, the reference level propagates at maximal velocity. The sites where the interface touches the reference height correspond to the active sites of DP. Therefore, in the active phase of DP the interface fluctuates close to the reference level so that the interface is smooth. On the other hand, in the inactive phase of DP, the interface detaches from the reference level and evolves into a rough state. More recently it has been shown that the critical behavior at the first few layers next to the reference height is equivalent to that of unidirectionally coupled DP processes [9].

DP itself is the generic universality class for phase transitions into absorbing states and covers a wide range of models. Only a few exceptions from DP are known.
One of them is the so-called parity-conserving (PC) class 10 15] which is represented most prominently by branching-annihilating random walks with even number of offspring (BAWE) 11. The PC class also includes models with two symmetric absorbing states 12, 14, 15, where the kinks between different absorbing domains may be interpreted as walkers whose number is preserved modulo 2. In other models 10,13 the two symmetric absorbing states emerge as checkerboard-like configurations of particles at even or odd sites, respectively.

Recently Park and Kahng [16] posed the question whether it is possible to replace the underlying DP mechanism in the interfacial growth models of Refs. [4] by a PC mechanism. To this end they introduced a model which involves two symmetric particle species and exhibits a roughening transition in $1+1$ dimensions. Since the dynamical rules at the bottom layer mimic a contact process with two symmetric absorbing states [15], the authors expected the roughening transition to be related to the PC class, in the same way as the transition in the monomer models of Ref. [5] is related to DP. But surprisingly the numerical estimates for the critical exponents deviated significantly from the PC values. Park and Kahng argued that the unexpected behavior may be related to the fact that dynamical processes at lower levels are strongly suppressed by particles at higher levels. In particular, kinks between different particle species may become frozen when they are covered by another layer of atoms.

In this Letter we introduce a model for dimer adsorption and desorption where this suppression effect is much less important. As dimers consist of two atoms, the number of particles at each height level is preserved modulo 2 . The dynamical rules are defined in a way that they mimic a BAWE at the bottom layer of the interface. It turns out that some of the critical properties of the roughening transition in this model are indeed characterized by PC exponents.

a. Definition of the model. The model is defined on a $d$-dimensional square lattice with $L^{d}$ sites and periodic boundary conditions. The interface height at site $i$ is 
$\mathrm{p}$
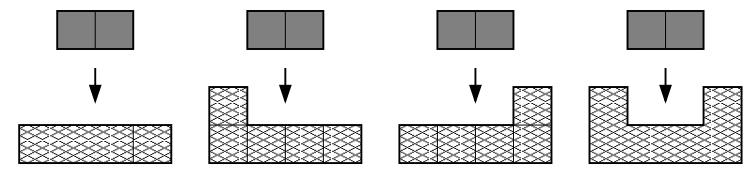

$1-\mathrm{p}$
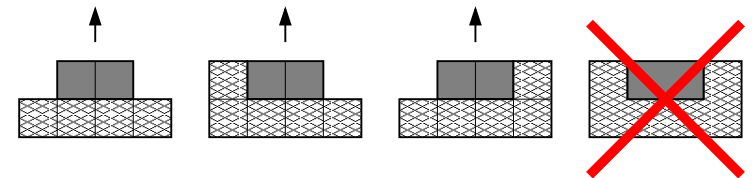

FIG. 1. Dynamical rules: Dimers are adsorbed with probability $p$ and desorbed at the edges of terraces with probability $1-p$.

represented by an integer height variable $h_{i}$ which obeys the restricted solid-on-solid (RSOS) condition

$$
\left|h_{i}-h_{j}\right| \leq 1, \quad|i-j|=1 .
$$

The model evolves by random sequential updates according to the following dynamical rules (see Fig. 1). In each attempted update two adjacent sites $i_{1}$ and $i_{2}$ are selected at random. If the heights $h_{i_{1}}$ and $h_{i_{2}}$ are equal, one of the following moves is carried out. Either dimers are adsorbed with probability $p$

$$
h_{i_{1}} \rightarrow h_{i_{1}}+1, \quad h_{i_{2}} \rightarrow h_{i_{2}}+1,
$$

or dimers desorb with probability $1-p$

$$
h_{i_{1}}, h_{i_{2}} \rightarrow \min _{k \in\left\langle i_{1}, i_{2}\right\rangle} h_{k},
$$

where $\min _{k \in\left\langle i_{1}, i_{2}\right\rangle} h_{k}$ denotes the minimum height of sites $i_{1}$ and $i_{2}$ and their nearest neighbors. An update will be rejected if it leads to a violation of the restriction (11).

The above model is defined in arbitrary spatial dimensions $d$ and is translationally invariant in space, time as well as in height direction. Moreover, it may be easily generalized to $n$-particle objects (monomers, dimers, trimers, etc.). In particular, for $n=1$ it reduces to the previously studied monomer growth model of Ref. [5] which is related to DP. We use random sequential updates in order to demonstrate that in contrast to PNG models [4,6], the phase transition in our model does not require synchronous updates. We have also studied another variant of the dimer model with parallel updates, as will be described elsewhere [17]. This cellular automaton model resembles a layer-by-layer crystal growth process and exhibits the same universal properties as the random-sequential variant.

b. Phenomenology of the roughening transition. If the adsorption rate $p$ is very small, only a few dimers are adsorbed, staying for a short time, before they quickly evaporate again. Therefore, the interface is smooth and anchored to the bottom layer (the spontaneously selected line of minimal height). As $p$ increases, more and more dimers cover the surface and large islands with several layers stacked on top of each other are formed. When $p$ exceeds a certain critical value $p_{c}$, the mean size of the islands diverges and the interface heights become uncorrelated over long distances, i.e., the interface evolves into a rough state.

In analogy to the monomer models of Ref. [5] we may expect the interface to detach from the bottom layer in the rough phase, resulting in a finite propagation velocity. However, it turns out that in the present case the interface remains pinned to the initial height and does not propagate at constant velocity. This is due to the fact that a stochastic deposition process cannot create a dense packing of dimers. The emerging configurations are rather characterized by a certain density of defects (solitary sites at the bottom layer) where dimers cannot be adsorbed. Because of the RSOS condition (11) these defects act as 'pinning centers' which prevent the interface from growing. The pinning centers cannot disappear spontaneously, rather they can only diffuse by interface fluctuations and recombine in pairs so that their number is expected to decrease extremely slowly. Therefore, the interface of an infinite system in the rough phase does not propagate at constant velocity. Instead the average height increases only logarithmically with time, as will be shown below.

In order to illustrate the PC mechanism in this model, let us interpret the sites at the bottom layer $h_{i}=0$ as $A$-particles. Adsorption and desorption processes correspond to certain effective reactions of the $A$-particles. For example, the adsorption of dimers at the bottom layer corresponds to a pair-annihilation process $2 A \rightarrow \varnothing$ at rate $p$. On the other hand, when dimers evaporate, two $A$-particles are created. However, since dimers can only evaporate at the edges of terraces, the presence of another neighboring $A$-particle is required, giving rise to an effective reaction $A \rightarrow 3 A$ at rate $1-p$. These two processes compete one another and realize a BAWE. This mapping is, of course, not exact since the $A$-particles are also coupled to the dynamical processes at higher levels of the interface. Nevertheless it seems that this feedback does not affect the critical behavior of the bottom layer.

c. Critical properties of the interface width. The order parameter that describes a roughening transition is the interface width

$$
W(t)=\left[\frac{1}{L^{d}} \sum_{i} h_{i}^{2}(t)-\left(\frac{1}{L^{d}} \sum_{i} h_{i}(t)\right)^{2}\right]^{1 / 2} .
$$

In order to investigate its critical properties, we perform Monte-Carlo simulations in $d=1$ dimensions on a lattice with $L=4096$ sites up to almost $10^{5}$ time steps. Starting from an initially flat interface $h_{i}=0$ we find the following results. Below the critical threshold $p<p_{c}$ the width saturates at some finite value which means that the heights $h_{i}$ are correlated over long distances. At the 

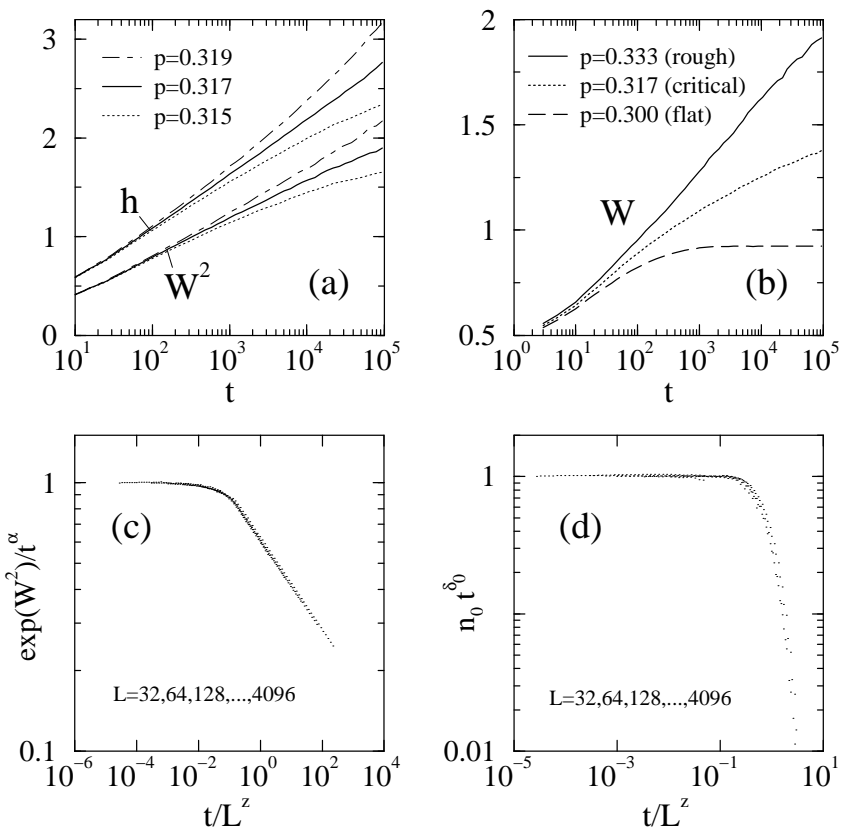

FIG. 2. Critical properties of the interface. In parts (a) and (b) the (squared) width and the average height are shown as functions of time near and far away from criticality, respectively. Parts (c) and (d) show data collapses for finite-size simulations (see text).

roughening transition $p=p_{c} \simeq 0.317(1)$ we observe that the squared width and the average height grow logarithmically with time as (see Fig. 2a)

$$
W^{2}(t) \sim \bar{h}(t) \sim \log t .
$$

In the rough phase $p>p_{c}$ this temporal behavior crosses over to $W(t) \sim \log t$ (see Fig. 2 $b$ ).

Assuming that the roughening transition is governed by an underlying PC transition, it is near at hand to conjecture the finite-size scaling form

$$
W^{2}(L, t) \simeq \log \left[t^{-\alpha} F\left(t / L^{z}\right)\right]
$$

where $F$ is an universal scaling function and $z=\nu_{\|} / \nu_{\perp}=$ $1.76(5)$ is the dynamical scaling exponent of the $\mathrm{PC}$ class. The exponent $\alpha$ is unknown and plays the role of a roughening exponent. In order to verify this scaling form, we perform finite-size simulations at criticality and plot $\exp \left(W^{2}\right) / t^{\alpha}$ for $10^{2} \leq t \leq 10^{5}$ against $t / L^{z}$. Using the estimate $\alpha=0.172(10)$ we obtain a fairly accurate data collapse (see Fig. 2 2 ), supporting that the dynamical exponent in this model is the same as in PC transitions.

d. Critical properties of the first few layers. We now turn to the critical behavior at the first few layers of the interface. Let us denote by

$$
n_{k}=\frac{1}{L^{d}} \sum_{j=0}^{k} \sum_{i} \delta_{h_{i}, j}, \quad k=0,1,2, \ldots
$$
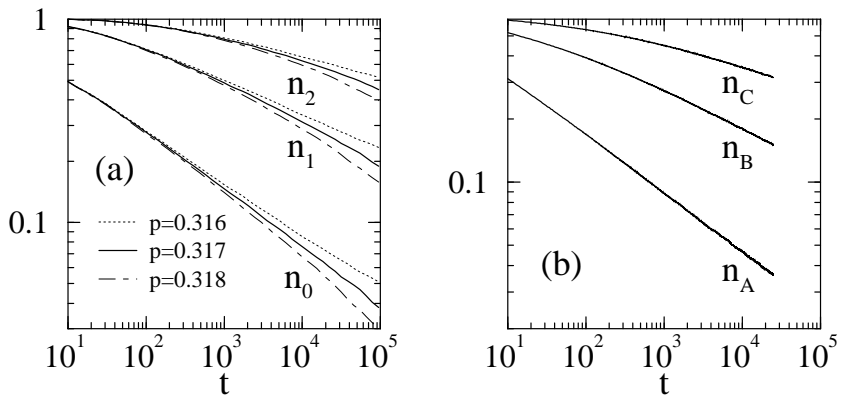

FIG. 3. (a) Densities $n_{k}$ at the first three levels as a function of time at and close to criticality. (b) Particle densities in unidirectionally coupled BAWE's at criticality.

the density of sites $i$ with $h_{i} \leq k$. In analogy to Ref. [5] we expect $n_{k}$ to obey the finite-size scaling form

$$
n_{k}(L, t) \sim t^{-\delta_{k}} G\left(t / L^{z}\right),
$$

where $G$ is a universal scaling function. $\delta_{0}=\delta=\beta / \nu_{\|}$ denotes the usual cluster survival exponent of the PC class while the other exponents $\delta_{1}, \delta_{2}, \ldots$ should be independent and smaller than $\delta_{0}$. Starting from a flat interface at $h=0$ in a large system with 4096 sites, we measure $n_{0}, n_{1}$ and $n_{2}$ at criticality (see Fig. 3a) which should decay as $n_{k}(t) \sim t^{-\delta_{k}}$. Averaging the slopes over the last decade in time we obtain the estimates

$$
\delta_{0}=0.280(10), \delta_{1}=0.200(15), \delta_{2}=0.120(15) .
$$

The estimates for $\delta_{0}$ and $z$ can also be verified by a finitesize data collapse (see Fig. 2d). The numerical value of $\delta_{0}$ is in fair agreement with the PC exponent $\delta=0.285(5)$, confirming that the dynamical processes at the bottom layer belong to the PC universality class.

e. Unidirectionally coupled BAWE's. In order to explain the exponents $\delta_{1}$ and $\delta_{2}$ we propose that the critical behavior at the first few layers in the dimer model should be equivalent to that of a hierarchy of unidirectionally coupled PC processes, just in the same way as the monomer model of Ref. [5] is related to unidirectionally coupled DP processes [9]. To this end we extend the particle interpretation: assuming that $h=0$ is the bottom layer of the interface, let us interpret sites $i$ with $h_{i} \leq 0,1,2, \ldots$ as particles $A, B, C, \ldots$, respectively. By definition, particles of different species are allowed to occupy the same site simultaneously. As shown before, the temporal evolution of the $A$-particles resembles a BAWE. Similarly, the $B$-particles perform an effective BAWE on top of inactive islands of the $A$-system. Clearly, the temporal evolution of the $B$-particles depends strongly on the dynamics of the $A$-particles. For example, $A$-particles instantaneously create $B$-particles at the same site, which may be interpreted as an effective reaction $A \rightarrow A+B$ at infinite rate. As this reaction does not modify the configuration of the $A$-particles, it couples the two subsystems only in one direction without feedback. On the 


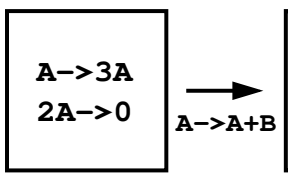

A

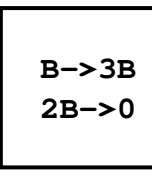

B

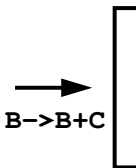

$\mathrm{C} \rightarrow 3 \mathrm{C}$ $2 \mathrm{C}->0$

C
FIG. 4. Unidirectionally coupled BAWE's.

other hand, the RSOS condition (1) introduces an effective feedback so that the $A$-particles are not completely decoupled from the $B$-particles. However, we will assume that in contrast to Ref. 16 this feedback is irrelevant for the critical behavior and can be neglected. Similarly, the $C$-particles are coupled to the $B$-particles by the effective reaction $B \rightarrow B+C$. Therefore the dimer model resembles a linear sequence of BAWE's which are effectively coupled without feedback in one direction, as sketched in Fig. 4 .

To verify this conjecture, we study three unidirectionally coupled BAWE's by Monte Carlo simulations (details will be published in [17]). We implement the coupling between the systems in a way that $A$-particles instantaneously create $B$-particles at the same site in the $B$-system, provided that this site is empty. Similarly, $B$-particles create $C$-particles at the same site. We simulate a hierarchy of three coupled BAWE's on a lattice of 2500 sites up to 25000 time steps and measure the densities of $A, B$, and $C$-particles as functions of time averaged over 1000 independent realizations (see Fig. $3 \mathrm{~b}$ ). It turns out that the particle densities $n_{A}, n_{B}, n_{C}$ decay in the same way as $n_{0}, n_{1}, n_{2}$ in the dimer model. Averaging the slopes over the last decade in time we obtain the exponents $\delta_{A}=0.280(5), \delta_{B}=0.190(10)$, and $\delta_{C}=0.120(15)$, which are in fair agreement with the corresponding exponents of the dimer model in Eq. (9). This supports the hypothesis that the dimer model and coupled BAWE's exhibit the same type of critical behavior.

It should be noted that the densities at higher levels do not scale perfectly. Rather the curves for $k \geq 2$ in Fig. 3 appear to be slightly bent, indicating a deviation from perfect scaling. This curvature is neither related to errors in the estimation of $p_{c}$ nor to finite-size effects. Similar deviations were also observed in the case of DP-related growth models 9 and are presumably caused by certain infrared divergent diagrams of the underlying field theory.

To summarize, we have introduced an interface model for dimer adsorption and desorption and have presented numerical evidence that the roughening transition in this model may be associated with the universality class of unidirectionally coupled PC processes.

Acknowledgements: The simulations were performed partially on the FUJITSU AP-3000 and System-V par- allel supercomputers. G. Ódor gratefully acknowledges support from the Hungarian research fund OTKA (Nos. T025286 and T023552).

[1] P. Meakin, in Phase Transitions and Critical Phenomena, ed. by C. Domb and J.L. Lebowitz (Academic, New-York, 1988), vol 12, p. 355.

[2] J. Krug and H. Spohn, in Solids far from Equilibrium: Growth, Morphology and Defects, ed. C. Godrèche (Cambridge University Press, Cambridge, 1990); A.L. Barabási and H.E. Stanley, Fractal Concepts in Surface Growth (Cambridge University Press, Cambridge, 1995); J. Krug, Adv. Phys. 46, 139 (1997).

[3] M. Kardar, G. Parisi, and Y.-C. Zhang, Phys. Rev. Lett. 56, 889 (1986); for reviews, see Ref. [2] and T. HalpinHealy and Y.-C. Zhang, Phys. Rep. 254, 215 (1995).

[4] J. Kertész and D.E. Wolf, Phys. Rev. Lett. 62, 2571 (1989); C. Lehner, N. Rajewsky, D.E. Wolf, and J. Kertész, Physica A 164, 81 (1990); A. Toom, J. Stat. Phys. 74, 91 (1994); J. Stat. Phys. 74, 111 (1994).

[5] U. Alon, M.R. Evans, H. Hinrichsen and D. Mukamel, Phys. Rev. Lett. 76, 2746 (1996); Phys. Rev. E 57, 4997 (1998).

[6] J.M. López and H.J. Jensen, Phys. Rev. Lett. 81, 1734 (1998).

[7] W. Kinzel, in Percolation Structures and Processes, ed. G. Deutscher, R. Zallen, and J. Adler, Ann. Isr. Phys. Soc. 5 (Adam Hilger, Bristol, 1983), p. 425.

[8] P. Grassberger and K. Sundermeyer, Phys. Lett. 77 B, 220 (1978). J.L. Cardy and R.L. Sugar, J. Phys. A 13, L423 (1980); H.K. Janssen, Z. Phys. B 42, 151 (1981).

[9] U.C. Täuber, M.J. Howard, and H. Hinrichsen, Phys. Rev. Lett. 80, 2165 (1998); Y.Y. Goldschmidt, Phys. Rev. Lett. 81, 2178 (1998); Y.Y. Goldschmidt, H. Hinrichsen, M.J. Howard, and U.C. Täuber, condmat/9809166).

10] P. Grassberger, F. Krause, and T. von der Twer, J. Phys. A 17, L105 (1984); P. Grassberger, J. Phys. A 22, L1103 (1989).

[11] H. Takayasu and A. Yu Tretyakov, Phys. Rev. Lett. 68, 3060 (1992); D. Zhong and D. ben-Avraham, Phys. Lett. A 209, 333 (1995); J.L. Cardy and U.C. Täuber, Phys. Rev. Lett. 77, 4780 (1996); J. Stat. Phys. 90, 1 (1998).

[12] N. Menyhárd J. Phys. A 27, 6139 (1994); N. Menyhárd and G. Ódor J. Phys. A 29, 7739 (1996).

[13] M.H. Kim and H. Park, Phys. Rev. Lett. 73, 2597 (1994); H. Park, M.H. Kim, and H. Park, Phys. Rev. E 52, 5664 (1995).

[14] K. E. Bassler and D. A. Browne, Phys. Rev. Lett. 77, 4094 (1996); Phys. Rev. E 55, 5225 (1997); K. S. Brown, K. E. Bassler, and D. A. Browne, Phys. Rev. E 56, 3953 (1997).

[15] H. Hinrichsen, Phys. Rev. E 55, 219 (1997).

[16] S. Park and B. Kahng, cond-mat/9807193.

[17] H. Hinrichsen and G. Ódor, (unpublished) 